\title{
Improving Production of Zebra Fish Embryos in the Lab
}

\author{
Robert Ohene Adu, Jens Peter Thomsen \\ Eco-Toxicology Research Group, Esbjerg Institute of Technology (EIT), Aalborg University, Esbjerg, Denmark. \\ E-mail: bertoadu@yahoo.com
}

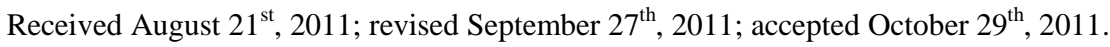

\begin{abstract}
The utilization of fish embryos in toxicity testing of hazardous chemicals has recently been adopted in order to satisfy stricter rules and regulations related to using adult animals in toxicity testing. This paper presents optimising steps towards improving zebra fish embryo production in the laboratory. Culture conditions were maintained in the aquaria as stipulated in the OECD draft proposal for a new guideline on fish embryo tests. Furthermore, a sequence of steps were adopted and followed to improve upon previous work done in the lab in 2006. About 200 eggs were produced in one spawn trap within an hour of onset of light, an improvement over the 50 - 60 eggs produced in the previous work. This result demonstrates that with the right culture conditions and proper optimisation of procedure the required number of embryos needed for toxicity testing can be obtained.
\end{abstract}

Keywords: Hazardous Chemicals, Zebra Fish Embryo, Toxicity Testing, Spawn Trap, Culture Conditions

\section{Introduction}

The use of juvenile and adult fish in toxicity testing of chemicals and effluents has been a conventional practice in eco-toxicology worldwide.

A number of factors render the use of fish suitable and important in eco-toxicity and environmental risk assessment. Some of these factors are:

- $\quad$ accidental fish kills are visible when they occur and raise public concern about the need to protect natural waters from pollutants;

- $\quad$ fish are considered sentinels for the drinking quality of waters used by man;

- the aquatic environment is a sink for many chemicals and this is evidenced by the occasionally high pollution levels and frequent chemical spills;

- fish play a critical role in aquatic food webs by regulating top-down and bottom-up flow of nutrient and energy;

- $\quad$ fish are an important source of food for humans;

- fishing has a large recreation value in many cultures.

In Europe toxicity testing with fish is done in order to regulate amounts of chemical substances in line with the goals of REACH (Registration, Evaluation, Authorization and restriction of Chemicals), under the EU Chemicals Agency, (ECHA) [1]. The increased production of chemicals on the market means that more and more fish have to be killed or poisoned for the purpose of establishing lethal and sub lethal concentrations.

As of 2005, experts predicted an additional 1.5 million animal tests needed to be carried out on about 30,000 existing chemicals in order to fully implement the objecttives of REACH, [2]. The establishment of REACH in 2007 has eliminated the distinction between existing substances and new substances in the EU market and has replaced some 40 EU Directives and Regulations [3,4]. This has meant that these 30,000 existing substances with annual market volumes of more than 1 ton, especially those marketed above 100 tons per year, will need to be tested under new toxicity testing schemes and their risk to human health and the environment will be assessed $[3,5,6]$. Out of these, some 140 chemicals have been given special attention by EU member countries and are to undergo comprehensive risk assessment [7].

At the same time, the continuous use of juvenile and adult fish in ecotoxicology work has come under attack from animal welfare groups for ethical, economic and scientific reasons. It is argued that in these bio-assays the primary endpoint is mortality and fish usually suffer pain or distress [7]. This, coupled with Directive 86/609/EEC regulating the use of animals in scientific experiments has provided the impetus for a reverse in the trend in fish use for toxicity testing. Thus an alternative testing pro- 
cedure using the embryos of fish instead in toxicity testing, called the fish embryo test (FET) has emerged. The embryonic development in relation to toxic concentration is registered during a test period of 48 - 96 hours. In Germany, for example, FET has replaced the conventional fish test since 2005 as an obligatory requirement for routine whole effluent testing (WET) and the test protocol is amenable to changes to make it suitable for chemical testing as well [8].

Scientific evidence strongly suggests that toxicity testing of chemicals with fish embryos is neither better nor worse than testing with adult fish. Results from a comparative re-evaluation of both fish embryo and adult fish toxicity data carried out on 143 substances showed a strong correlation between the two [7]. Indeed, using the zebra fish as an example, a comparison of 21 data pairs of lethal concentrations that kill $50 \%$ of the population $\left(\mathrm{LC}_{50} \mathrm{~s}\right)$ carried out on the adult fish and the embryo showed a regression with a correlation of 0.81 and a slope of 1.12 [7].

The Zebra fish (Danio rerio sp.), a tropical freshwater fish has become a model in ecotoxicology and environmental sciences with a wide range of future applications for integrative risks assessment of chemicals [9]. The species is native to the streams of Southeastern Asia, especially Nepal, India, Pakistan, Bangladesh among others. It has been noted to possess sterling qualities such as small size of the species, its cheap and easy husbandry, superb fertility, production of transparent embryos, rapid in-vitro development of the embryos and simple, accurate chemical delivery [10].

This study, as a prelude to the embryo assay, looked at steps towards enhancing the production of embryos of the Zebra fish (Danio rerio sp.) for use in the fish embryo toxicity testing. It is aimed at developing an appropriate methodology towards maximising the production of embryos of the zebra fish in the lab. This test has been proposed for testing the toxicity of a number of chemicals such as saponins, speculated to be responsible for recent fish kills in a small stream in Denmark [11]. Saponins are naturally-occurring glycosides considered to be present in horse chestnuts (Aesculus hippocastanum sp.). They are known to cause hemolysis of red blood cells in fish [10].

Previous work done in the laboratory by [12] saw iregular egg production and therefore inadequate embryos for a thorough test. When eggs were produced they were usually between 50 and 60 , less than the number required for a duplicate test. As a result there is need for a standardized procedure to maximize egg production for the series of tests required. According to the procedure outlined in the OECD Draft guideline for Fish Embryo Toxicity test [13], the test should be performed in 2 - 3 replicates, each hav- ing at least 5 concentrations of the test substance and a control group in 24-well plates. This requires a minimum of 120 embryos to meet the statistical requirements. To achieve this, a number of optimising steps were needed to ensure this production.

\section{Materials and Methods}

\subsection{Experimental Set-Up}

The set-up involved 2 aquaria (Waterhome) each of $40 \mathrm{~L}$ capacity and a maintenance tank to maintain an optimum water level. A breeding stock of mature zebra fish (Danio rerio sp.) of about 6 months were used. Each aquarium had 20 fishes ( 13 males and 7 females) for adequate spawning and fertilization. To maintain a suitable temperature range of $26^{\circ} \mathrm{C} \pm 1^{\circ} \mathrm{C}$ for the zebra fishes, two $50 \mathrm{~W}$ heaters (Elite $50 \mathrm{~W}$ ) were installed on the aquaria. Two lamps (Sun-Glo A1590) were fitted inside the aquaria and connected to a clock timer to regulate a light-dark regime of 14 hours light and 9 hours darkness. As aquarium water, suitable dilution water with oxygen saturation at $26^{\circ} \mathrm{C} \pm 1^{\circ} \mathrm{C}$ not less than $80 \%$ was used for breeding the zebra fish [13]. Oxygen was supplied through pumps installed inside the two aquaria. The dissolved oxygen concentration was measured by means of an OxyTop Dissolved Oxygen (D.O) Probe. The pH of the aquarium water was measured with a standard $\mathrm{pH}$ Meter (Meter Lab pH M210) and ensured to be between 6.8 and 8.4 [13]. Dry flaky food was supplied about 3 times a day to the fishes through a programmable fish-feeding timer (Nutrafin ProFeed Plus) installed on each aquarium whilst live Artemia species were manually administered once a day to promote breeding. The fishes require a combination of both flakes and live foods to ensure food variety. In their natural waters zebra fish feed on smaller organisms such as zooplanktons. Care was how- ever taken not to over feed the fish.

A spawn trap was placed inside each aquarium to collect the eggs spawned. To separate these spawned eggs from their mother fishes, each spawn trap was covered with a stainless steel grid of mesh size about $1.25 \mathrm{~mm}$, big enough to let the eggs drop through. Fastened to the grid on the spawn trap were artificial plants made of plastic to stimulate breeding. Each spawn trap was placed on an artificial green plastic carpet inside the aquarium to serve as substrate (Figure 1).

Photo-resist curtains were fitted around the aquaria to ensure that during the 9 hours when the lamps were timed off at night the zebra fishes experienced complete darkness.

\subsection{Methodology}

For the culture conditions in the aquaria, the temperature 


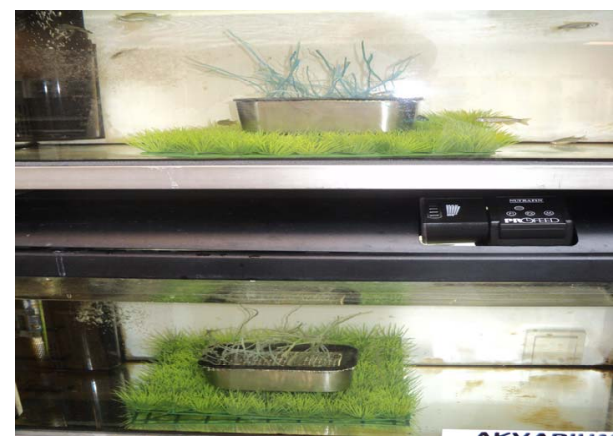

Figure 1. Aquaria with spawn traps.

was set at $26^{\circ} \mathrm{C} \pm 1{ }^{\circ} \mathrm{C}$, whilst a photoperiod of $14 \mathrm{hr}$-light: $8 \mathrm{hr}$-dark periods was set ( 9 am to $11 \mathrm{pm}$ light). To ensure this, the photo-resist curtains were drawn to cover the aquaria before leaving lab to ensure that the fishes experienced complete darkness from $11 \mathrm{pm}$ when the light was timed off until the next morning at $9 \mathrm{am}$. Zebra fish were bred at a male-to-female ratio of 2:1 ( 27 males and 13 females). Since fertilization in zebra fish is in-vitro there is need for twice as many males as females to ensure that enough sperms are spawned to fertilize the spawned eggs.

The two spawn traps were gently removed before 9 am and emptied of any old eggs. Old eggs were transferred into Petri dishes and the traps were quickly put back inside the aquaria and covered with the curtain again before onset of light. One hour after onset of light (10 am) the spawn traps were removed again and fresh eggs (1hour old) were transferred into separate petri dishes. The live fish food, Artemia, was now introduced. This was done once a day to promote breeding. When this food had settled down in the aquaria, the spawn traps were put back. This was to ensure that the spawn traps were always free of Artemia food. Fertilized eggs were identified under a stereo microscope and counted.

The physico-chemical conditions in the aquaria namely dissolved oxygen (D.O) level, $\mathrm{pH}$ and temperature were determined using the D.O probe and $\mathrm{pH}$ meter respecttively.

The dry flakes food was periodically introduced about 3 times a day by the automatic feeding machine installed with a timer [13].

\section{3. pH and Dissolved Oxygen}

The $\mathrm{pH}$ of the maintenance water inside the two aquaria were 8.34 and 8.30 whilst dissolved oxygen levels were $7.4 \mathrm{mg} / \mathrm{L}$ representing more than $80 \%$ saturation which meet the OECD requirements (Table 1).

\section{Results and Discussion}

About 200 embryos were obtained in one spawn trap
Table 1. Physicochemical conditions inside aquaria.

\begin{tabular}{ccc}
\hline & Aquarium 1 & Aquarium 2 \\
\hline $\mathrm{pH}$ & 8.34 & 8.3 \\
Dissolved Oxygen & $7.4 \mathrm{mg} / \mathrm{L}(94 \%)$ & $7.4 \mathrm{mg} / \mathrm{L}(94 \%)$ \\
Temperature & $27.4^{\circ} \mathrm{C}$ & $26.5^{\circ} \mathrm{C}$ \\
\hline
\end{tabular}

within one hour of onset of light (Figure 2). This was an improvement over the previous work which recorded 50 - 60 eggs in an aquarium [12]. Thus newly-formed embryos less than 2 hours old (1-cell stage, Figure 3) were available for toxicity testing with any chemical or effluent to be used. The sensitivity of embryos to chemical or effluent exposure has been shown to depend on embryo age at start of exposure. In an experiment testing, for example, 2,4 dinitrophenol on zebra fish embryos, it was clearly demonstrated that eggs 8 hours and older showed no mortality at all after 48 hours of exposure [8].

A sensitivity study carried out by Lahnsteiner, 2008 [14] also confirmed the importance of using early onto-genetic stages of embryo for the test.

\section{Conclusions}

It is thus possible, from the results obtained, that at least

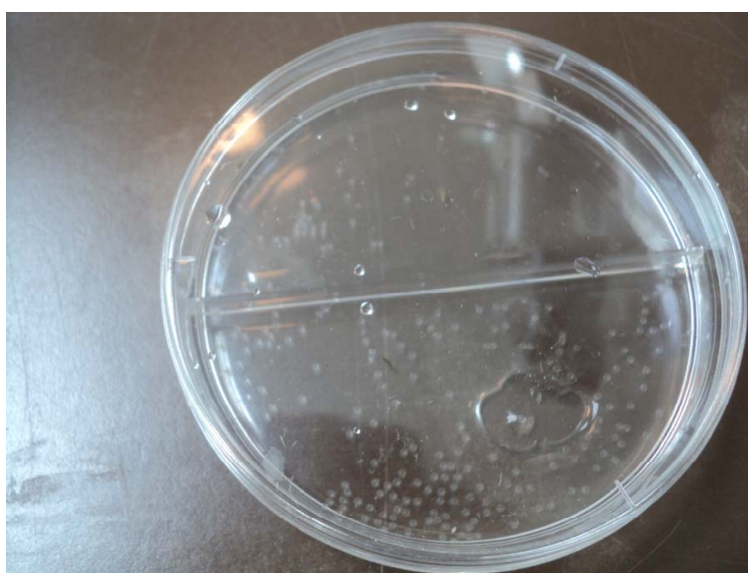

Figure 2. Newly-fertilized eggs in Petri dish.

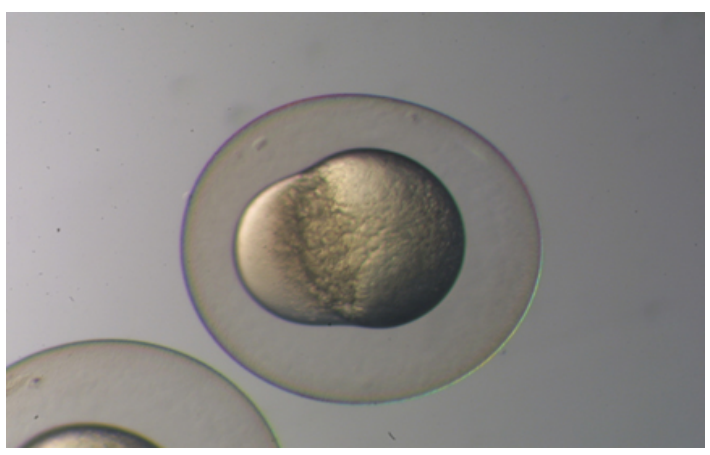

Figure 3. Embryo at 1-cell stage under microscope. 
200 eggs can be obtained in each aquarium within 1 hour of onset of light, provided that optimal culture conditions and the right procedural steps as indicated in this study are followed. Slight modifications in procedure may be necessary as per the peculiar conditions obtaining in the lab.

\section{Acknowledgements}

The authors would like to thank the section leader, Eric Sogaard, for his approval for this research to be undertaken. Especial thanks also go to the lab technician, Linda Madsen, for her immense support in helping to carry out the laboratory work.

\section{REFERENCES}

[1] S. Scholz, S. Fischer, U. Guundel, E. Kuuster, T. Luckenbach and D. Voelker, "The Zebrafish Embryo Model in Environmental Risk Assessment-Applications beyond Acute Toxicity Testing," Environmental Science and Pollution Research, Vol. 15, No. 5, 2008, pp. 394-404.

[2] G. Schüürmann, K. Schirmer, E. Küster, B. Hansjürgens and W. Köck, "Using REACH as an Opportunity to Find Alternatives to Animal Experiments: A Need for Intelligent Test Strategies Instead of Blindly Testing Everything,” 2005. http://www.ufz.de/index.php?en=6556

[3] V. J. Brown, “Reaching for Chemical Safety,” 2003. http://www.ncbi.nlm.nih.gov/pmc/articles/PMC1241736/ pdf/ehp0111-a00766.pdf

[4] T. Petry, R. Knowles and R. Meads, "An Analysis of the Proposed REACH Regulations,” 2006. http://toxminds.org/pdf_publications/TP-REACH-RTP-2 006.pdf

[5] R. Combes, M. Baratt and M. Balls, “An Overall Strategy for the Testing of Human Hazard and Risk Assessment under the EU REACH System,” Alternatives to Laboratory Animals, Vol. 31, No. 1, 2003, pp. 7-19.

[6] W. Lilienblum, W. Dekant, H. Foth, T. Gebel, J. G. Hengstler, R. Kahl, P. J. Kramer, H. Schweinfurth and K. M. Wollin, “Alternative Methods to Safety Studies in Expe- rimental Animals: Role in the Risk Assessment of Chemicals under the New European Chemicals Legislation (REACH),” 2008. http://www.ncbi.nlm.nih.gov/pubmed/18322675

[7] E. Lammer, G. J. Carr K. Wendler; J. M. Rawlings, S. E. Belanger and Th. Braunbeck, "Is the Fish Embryo Toxicity Test (FET) with the Zebra Fish (Danio rerio) a Potential Alternative for the Fish Toxicity Test?” Comparative Biochemistry and Physiology Part C: Toxicology \& Pharmacology, Vol. 149, No. 2, 2009, pp. 196-209. doi:10.1016/j.cbpc.2008.11.006

[8] T. Braunbeck and E. Lammer, "Background Paper on Fish Embryo Toxicity Assays. Prepared for German Federal Environment Agency,” UBA Contract No. 20385 422, 2006.

[9] K. Schirmer, K. Tanneberger, N. I. Kramer, D. Völker, S. Scholz, C. Hafner, E. J. Lee, N. C. Bols and J. L. M. Hermens, "Developing a List of Reference Chemicals for Testing Alternatives to Whole Fish Toxicity Tests," 2008. http://www.wlu.ca/documents/32518/Schirmer_et_al_aqu a_tox08.pdf

[10] S. M. Hassan and E. A. Moussa, "Effects of Quillaja Saponin (Quillaja saponaria) on Early Embryonic Zebrafish (Danio rerio) Development,” International Journal of Toxicology, Vol. 27, No. 3, 2008, pp. 273-278. doi:10.1080/10915810802152129

[11] S. B. Frandsen, “Chestnuts Cause Fish Kills,” 2010. http://www.kolding.dk/data/0067955.asp?sid=13552\&uid $=24949$

[12] M. Wirtz, “Toxicity and Possible Biodegradation of Groundwater from a Former Chemical Dump Site (Kaaergard Plantage)," University of Applied Sciences Oldenburg/Ostfriesland/Wilhelmshaven; Aalborg University, Esbjerg, 2006.

[13] OECD, "OECD Guideline for the Testing of Chemicals," Draft Proposal for a New Guideline: Fish Embryo Toxicity (FET) Test, 2006.

[14] F. Lahnsteiner, "The Sensitivity and Reproducibility of the Zebrafish (Danio rerio) Embryo Test for the Screening of Waste Water Quality and for Testing the Toxicity of Chemicals," ATLA. Alternatives to Laboratory Animals, Vol. 36, No. 3, 2008, pp. 299-311. 